\title{
Artificial Intelligence in the Field of Electrical Engineering
}

\author{
Sesha Gopal S \\ Dept. of EEE \\ BMS Institute of Technology \& Management \\ India
}

\begin{abstract}
This Electricity runs the world today. It is also noteworthy that the world today will not run function if it is deprived of electricity. A power system can be considered as an interconnection of electrical components that are used to utilize the power in an effective way and help in the transfer and supply of the power to the consumers. There are a lot of aspects that need to be considered while manufacturing components for an electric power system as well as the maintenance of the said power system. There is a possibility of human error that can arise during the maintenance and manufacturing of parts as well as ensuring the security of the system. A possible solution to this comes in the form of artificial intelligence. Artificial intelligence can be visualized as an effort to emulate the ability of a human being to think rationally and process the information available in order to reach conclusive results. This paper aims to study more about what artificial intelligence is and how it can be used in the field of electrical engineering.
\end{abstract}

Keywords-Artificial intelligence, electrical engineering, power systems, neural networks, fuzzy logic.

\section{INTRODUCTION}

John McCarthy coined the term Artificial Intelligence in the year 1956 and defines it as "The science and engineering of making intelligence machines". This field of study came into existence on the assumption that human intelligence can be described so precisely that it can be simulated by a machine. Artificial intelligence is an emerging technology that not only studies the theory, technology and the application systems for simulating and extending human intelligence, but also develop all of the above, involving various disciplines such as psychology, cognitive science, thinking, information science, system science and bio science.

Artificial intelligence has provided a great potential and space for optimization in the field of electrical engineering. This can not only bring about a significant improvement not only with regards to the economic aspects but also in the safety and the actual control of the operation. Ever since artificial intelligence reached a stage where it could be used in various fields in order to make work simpler, it has been used widely in various fields of life, primarily in robotics and computer programming. Recent studies and investigations have shown that artificial intelligence can also be a viable option in the field of electrical engineering especially as a solution to some longstanding power system problems where conventional methods experience some difficulty.

\section{PROS AND CONS OF ARTIFICIAL INTELLIGENCE}

As an emerging technology, artificial intelligence has its fair share of advantages and disadvantages. By studying more about the pros and cons of artificial intelligence, we can decide on how artificial intelligence can be integrated with electrical engineering and power systems in order to facilitate a smarter system.

\section{A. Pros of artificial intelligence:}

- First, Reduction in human error: With the increase in amount of data being supplied, artificial intelligence can completely nullify human error and thereby give a much more accurate result when compared to the results provided by humans. This is a huge advantage as accuracy is very important in the field of electrical engineering as one single mistake can not only disrupt the power system but also lead to catastrophic results.

- Safety of human labor: Another major advantage of artificial intelligence is the fact that human beings do not have to directly interact with scenarios that can be risky or pose a threat to the labor. In case of a fire in an apartment, robots powered by AI can be used to navigate through the building and put out the fire, thereby nullifying the risk to human life as well as accomplishing the task with a satisfactory outcome.

- Repetition: There are a lot of tasks that need to be repeated in order to be completed. Tasks such as sending the same email to a group of people or installing a particular software on the system of each and every employee's computer system are considered to be boring and tedious. Artificial intelligence can be used in order to tackle such tasks with ease, thereby not only completing the task but also ensuring that human labor is not wasted on such tasks. A possible area of application can be in the manufacturing and automation of various electrical components.

- Speed of decision making: Since artificial intelligence is ultimately carried out by machines, it is generally faster. The main difference between artificial intelligence and human intelligence is that human beings analyze all the factors pragmatically as well as emotionally and then come to a decision based on what that person feels is right. Therefore, the decision can vary wildly from person to person. Artificial intelligence on the other hand gives an output considering all the factors based on how it has been programmed. This approach significantly speeds up the problem solving and decision making. This can prove to be extremely important in case of 
power outages and disruptions in the power delivery to the consumers.

- Availability: Problems can occur without a warning. It is imperative for a system to be able to tackle any issue that can crop up at any time. Human beings cannot be available $24 \mathrm{X} 7$ and in order to do so, people need to work in shifts to satisfy the need of supervision for the whole day. Artificial intelligence is available the whole day owing to the fact that it is a computer program that analyzes the various inputs being provided to the system. Robots and machines that make use of artificial intelligence do not tire like humans thereby increasing the cumulative hours of work put in as well.

Looking at the number of advantages that artificial intelligence can bring to the table might be enough to convince someone that it can be a viable option to be used in the field of electrical engineering. But it is also important to understand the shortcomings of artificial intelligence in order to implement innovative solutions to overcome them.

\section{B. Cons of artificial intelligence:}

- The Unemployment: This is one of the most controversial points of focus when it comes to whether artificial intelligence must be implemented in various industries. There is a fraction of people who believe work will be made easier with the adoption of artificial intelligence in a lot of fields but there are people who believe that artificial intelligence can replace human labour in these fields, thereby eliminating the need of humans. This can lead to unemployment and loss of pay to a lot of people who depend on this work in order to pay for their food and expenditure.

- Propagation of laziness: Artificial intelligence is not only available $24 \times 7$ but also does not tire and perform at peak efficiency all day. As discussed above, this eliminates the need for human supervision as well, thereby making us complacent and lazy as we feel that the artificial intelligence can deal with the problems at hand.

- Lack of emotional intelligence: As discussed earlier, artificial intelligence solves problems and makes decisions much faster than human beings but this comes at a cost. Humans think emotionally as well and a part of this goes into the decision-making procedure as well but artificial intelligence does not consider this into its decision-making. Artificial intelligence just does as programmed and programming of emotions will take a lot of study, research and effort from scientists all over the world.

- Cost: Integrating artificial intelligence into an already existing system not only consumes a lot of time and effort but also costs a lot of money. This means that there is a requirement for a capital amount that needs to be invested in order to make artificial intelligence a viable option.

- Time of implementation: Due to a lack of extremely skilled programmers and the difficulty in the development of a satisfactory model, developing the suitable artificial intelligence in order to tackle real life situations will take a lot of time to develop and test.

Now that we have a clear understanding of the various pros and cons of artificial intelligence, we can delve more on how to integrate artificial intelligence into electrical engineering and the various applications and problems that can be addressed as a result of this.

\section{AI IN ELECTRICAL ENGINEERING}

There are different types of artificial intelligence that can be applied to a modern-day power system and they are:

- Expert System Techniques.

- Artificial Neural Networks.

- Fuzzy Logic Systems.

A. Expert systems:

In simple terms, an expert system can be defined as a computer system that incorporates the decision-making ability of a human expert, which means these kinds of systems specialize in a particular field of study. This can also pose a problem as these systems cannot handle new problems or situations that can arise. Since expert systems are typically just computer programs, they can be used in order to calculate and determine parameters and values in the generation, transmission and the distribution of power.

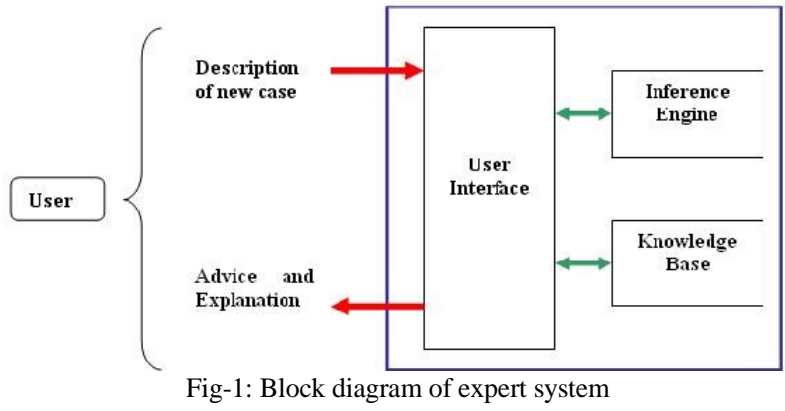

\section{B. Artificial Neural Networks:}

An artificial neural network is based on a collection of interconnected nodes or units called artificial neurons, which are actually modelled based on the biological neural networks present in the human body. The main goal of an ANN is to emulate the behavior of a human brain and thereby solve problems just like the human brain by employing various strategies such as decision-making and problem solving. There are various deviations of this such as computer vision and speech recognition.

ANN's basically convert a set of inputs into a corresponding set of outputs by passing the input through a network of neurons. Each neuron that is a part of the neural network produces its own output based on the input that is given to that neuron.

ANN's find their application in power system stabilizers, load forecasting, load modelling and state estimation. 


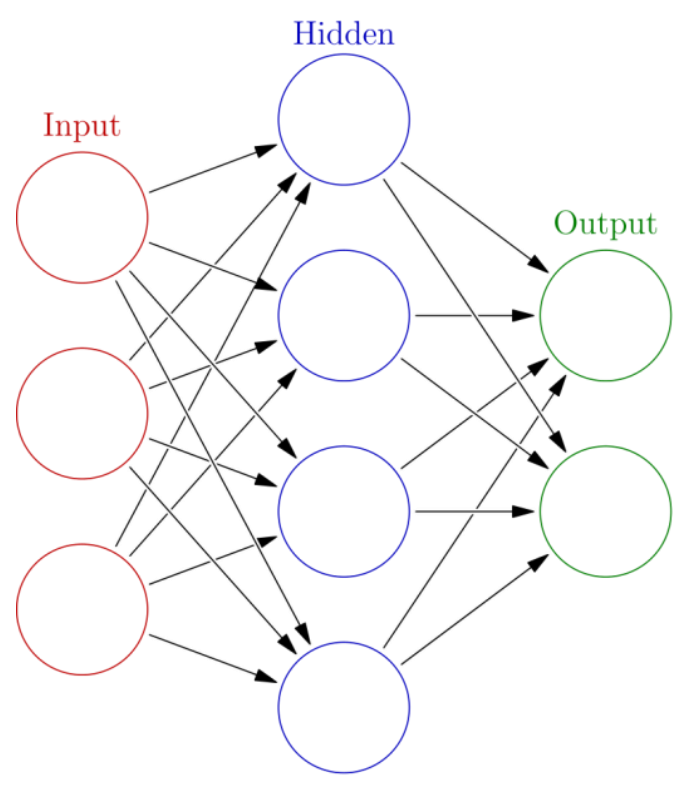

Fig-2: Block diagram of an ANN

C. Fuzzy logic systems:

Fuzzy logic can be visualized as a many-valued logic, as compared to Boolean logic which basically has just two values for the output i.e. 0 and 1. Fuzzy logic gives the output in the form of truth values that may be any real number between 0 and 1 as a way to incorporate the idea of partial truth. There may be some cases where the output can be completely true or completely false, but a majority of the cases have some degree of truth or falsehood coupled with them. This is based on the observation that people usually make decisions based on imprecise and non-numerical information, therefore the name fuzzy logic.

Fuzzy logic finds its application in the control of power systems like voltage control, stability control, power flow control, stability analysis and enhancement, load forecasting and performance improvement of transmission lines.

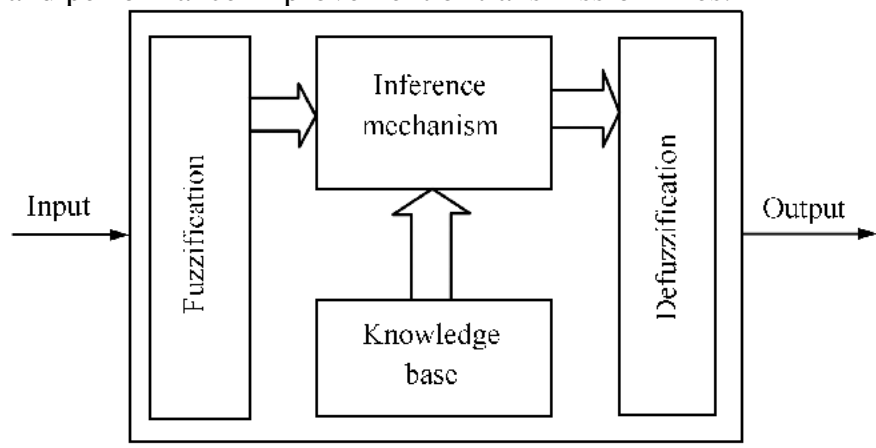

Fig-3: Block diagram of a fuzzy system

\section{APPLICATIONS OF AI IN ELECTRICAL ENGINEERING}

As discussed, several problems in power systems cannot be solved by conventional methods owing to the fact that the requirements for these methods to function are not feasible at that point in time. A few broad areas that artificial intelligence can possibly be used in are:

- Operation of power system: Unit commitment, hydro-thermal coordination, economic load dispatch, maintenance scheduling, and load and power flow.
- Planning of power system: Generation expansion planning, power system reliability, transmission expansion planning, and reactive power planning.

- Control of power system: Voltage control, stability control, power flow control, and load frequency control.

- Control of power plants.

- Automation of power system: Restoration, management, fault diagnosis, and network security.

- Distribution systems: Planning and operation of distribution systems, demand side response and management, operation and control of smart grids, and network configuration.

- Distributed generation applications: Distributed generation planning, solar photovoltaic power plant control, wind turbine plant control, and renewable energy resources.

- Forecasting: short term load forecasting, long term load forecasting, electricity market forecasting, solar power forecasting, and wind power forecasting.

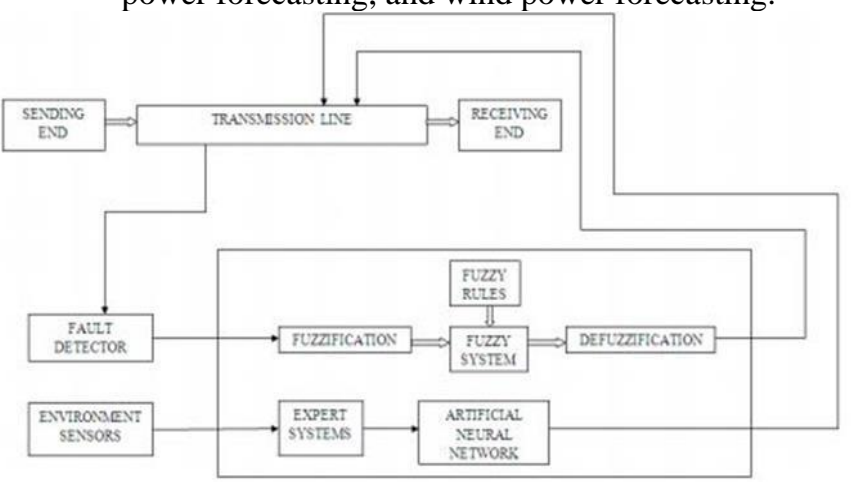

Fig-4: Fuzzy logic in a transmission line

Since electrical engineering is a highly specialized branch, it requires the use of expert systems in order to solve problems by employing decision making, archiving knowledge, and solving problems by the help of reasoning, judgement and heuristics. Fuzzy logic systems can primarily be used in fault diagnosis. Assume that a fault has occurred on the transmission line. The fault detector can feed the information of this fault into the fuzzy logic system. The fuzzy system then processes this information in order to give us a crisp output of what the fault is.

Artificial neural networks can be used in improving the performance of the transmission lines. Various sensors can be added to the transmission lines in order to constantly monitor the environment and other ambient conditions. These conditions can then be fed into the artificial neural network in order to process it and then change the line parameters in order to improve the performance of the line. The improvement in performance is directly proportional to the efficiency of the ANN. The efficiency and speed of operation of the ANN can be improved by increasing the number of hidden neuron layers in the ANN.

It is pretty evident how much of an impact artificial intelligence can have on power systems. The adoption of artificial intelligence will make our existing systems efficient and reliable. 


\section{CONCLUSION}

Artificial intelligence is an emerging field and the applications of AI in various fields of study only keeps growing day by day. Electrical power systems require a lot of deliberation when it comes to manufacturing, maintenance and security and this is where artificial intelligence comes into the picture. Artificial intelligence has its fair share of advantages and disadvantages but the impact that the advantages have on electrical systems as a whole far outweighs the disadvantages. The different types of artificial intelligence available and the various applications of artificial intelligence in electrical engineering have been discussed in detail. It can also be concluded that artificial intelligence is an extremely viable choice of technology that can be included in the field of electrical engineering in order to not only make life simpler but also bring in a high degree of efficiency and reliability into the system. A lot of research is required in order to fully incorporate AI into electrical engineering and a simply study of what $\mathrm{AI}$ is capable of and the variety of applications it can possibly be used in is a stepping stone in the right direction.

\section{REFERENCES}

[1] Advantages and disadvantages of artificial intelligence. Source : https://towardsdatascience.com/advantages-and-disadvantages-ofartificial-intelligence-182a5ef6588c

[2] C. Karthik, "Intelligence in electrical engineering", IJERT CONFCALL-2019.

[3] Mughal, Shafqat \& Malik, Hasmat. (2011). Application and Implementation of Artificial Intelligence in Electrical System.

[4] Bhagath Sivadasan, "Application of artificial intelligence in electrical engineering", GRDJE, National conference on Emerging Trend in Electrical and Electronics Engineering, March 2018.

[5] Han Feng, "The application of artificial intelligence in electrical automation control", Journal of Physics, Conf. Ser.1087 062008.

[6] Artificial Neural Networks. Source of the information : https://en.wikipedia.org/wiki/Artificial_neural_network

[7] Fuzzy logic: https://searchenterpriseai.techtarget.com/definition/fuzzylogic

[8] Wonohadidjojo, Daniel \& Kothapalli, G. \& Hassan, Mohammed, "Position Control of Electro-hydraulic Actuator System Using Fuzzy Logic Controller Optimized by Particle Swarm Optimization" International Journal of Automation and Computing. 